\title{
Opportunistic Scheduling over Wireless Fading Channels without Explicit Feedback
}

\author{
Mehrdad Dianati, Rahim Tafazolli \\ Centre for Communication Systems Research (CCSR) \\ Department of Electronics and Electrical Eng., University of Surrey \\ Guildford, GU2 7XH, Surrey, UK
}

\begin{abstract}
A novel approach for implementation of opportunistic scheduling without explicit feedback channels is proposed in this paper, which exploits the existing ARQ signals instead of feedback channels to reduce the complexity of implementation. Monte Carlo simulation results demonstrate the efficacy of the proposed approach in harvesting multiuser diversity gain. The proposed approach enables implementation of opportunistic scheduling in a variety of wireless networks, such as the IEEE 802.11, without feedback facilities for collecting partial channel state information from users.
\end{abstract}

\section{INTRODUCTION}

Effective channel resource management improves bandwidth utilization without degradation of Quality of Service (QoS) for network users. A typical resource management system in a wireless base station, as shown in Figure 1, incorporates three subsystems for: 1) access control to regulate the incoming traffic; 2) admission control to avoid system overload; and 3) scheduling for dynamic resources sharing among active users. The scheduling subsystem, which is the
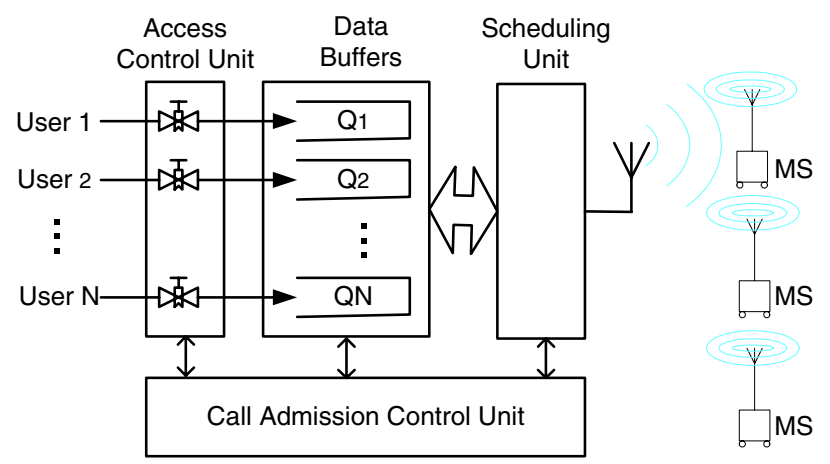

Fig. 1. A typical resource management system

focus of this paper, prioritizes transmission of delay tolerant data to multiple active users. The main objective of an effective scheduling scheme is in general to harvest statistical multiplexing gain while maintaining fairness among users. For wireless channels smart scheduling can also improve spectrum efficiency. In a scenario where a wireless base station with a single transmit antenna streams data to multiple users, when the partial Channel State Information (CSI) is available, the optimal scheduling strategy to maximize spectrum efficiency is to transmit to a single user with the best channel quality in each scheduling epoch (i.e., time slot) [1][2]. This can be considered as an opportunistic service discipline that relies on existence a feedback channels to collect partial CSI from users. Opportunistic scheduling with Adaptive Modulation and Coding (AMC) are widely proposed for modern wireless systems [3]-[7]. However, necessity of an explicit feedback channel is often criticized as a practical hinderance in real systems and a hidden spectrum cost [8]. Thus, elimination of explicit feedback channel appears to be an interesting proposition in this context.

Wireless channels are characterized by a high bit error rate such that relying on only forward error correction techniques is not efficient in order to reasonably improve link level reliability. Automatic Repeat reQuest (ARQ) schemes are therefore de facto parts of wireless link layer protocols for both the existing systems [9] and the new proposals [10]. In this paper, we propose implementation of opportunistic scheduling without explicit feedback channels relying on the existing ARQ messages. Limited information, learned from ARQ messages, is used to estimate the status of channel for each user. The estimated information is then used to make scheduling decisions. Monte Carlo simulations are used to demonstrate the efficacy of the proposed approach. The results indicate that our approach can significantly improve the total throughput of scheduling, maintain fairness among user and, reduce the complexity of implementation.

The rest of this paper is organized as follows. In Section II, the system model and the major assumptions are presented. The scheduling schemes that are studied in this paper will be presented in Section III. In Section IV, the simulation scenarios are specified and some sample performance analysis results are presented and discussed. The concluding remarks are given in Section V.

\section{SYSTEM MODEL}

The system model in this paper incorporates a single base station with multiple mobile stations as shown in Figure 2. The base station and each of the mobile stations are equipped with a single antenna. Downlink data streams for different users arrive at the base station and are placed in separate buffers for transmission. A central scheduler allocate an entire time slot of $1.25 \mathrm{~ms}$ to only one user at each time. Mobile stations send back a positive acknowledgment (ACK) upon successful reception of a data frame; otherwise, they either return a 
negative acknowledgment (NAK) or the base station assumes an implicit NAK after a certain time out period. Since the focus of this paper is to investigate the impacts of scheduling schemes on spectrum utilization, we consider a saturated case, where there is always backlogged data for transmission inside the data buffers.

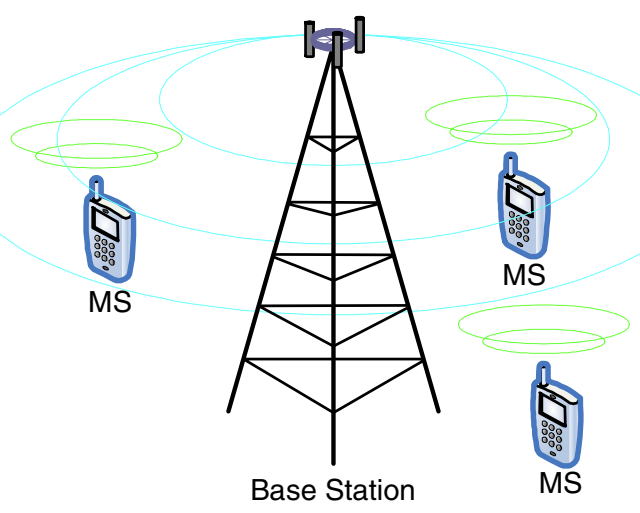

Fig. 2. System model

We consider a quasi static Rayleigh fading model for the wireless channels. It has been shown that the envelope of the received signal, $|g(t)|$, for this model demonstrates strong Markovian properties [12]. If the normalized received signal power, $|g(t)|^{2} / \Omega$ (note that $\Omega$ represents the average envelope power), is divided into a finite number of states, as shown the right side of Figure 3, a finite-state discrete Markov Process can adequately capture the dynamic of transition between different states. Note that in Figure 3, $\left\{\xi_{i}\right\}$ represents a set of finite thresholds and $\left\{p_{i, j}\right\}$ is a set of state transition probabilities which can be obtained by the proposed algorithm in Appendix .

If the noise plus interference power has Gaussian distribution, the channel in each states resembles an AWGN channel with different Signal to Noise and Interference Ratio (SINR). For AMC-based systems, having the CSI, the transmitter can adapt a proper transmission rate with a corresponding Modulation and Coding Scheme (MCS) to achieve a certain Packet Error Rate (PER), e.g, 1\%. The relationship between achievable data rate, MCS, and PER is usually complicated for practical systems; analytical models are often too complex or computationally prohibitive. Link level simulation results are widely used for performance analysis and implementation purposes. However, since in this paper we intend to compare the performance of scheduling schemes, we assume a perfect physical layer which can achieve Shannon's performance bound. Thus, for an actual SINR value, $S I N R_{a}$, if the transmission rate, $R_{t}$ is below $R_{a}=B \log _{2}\left(1+S I N R_{a}\right)$ the packet error rate is zero. If the estimated SINR value, $S I N R_{e}$ is less than the actual SINR value, according to strong inverse coding theorem the PER will approach to one.

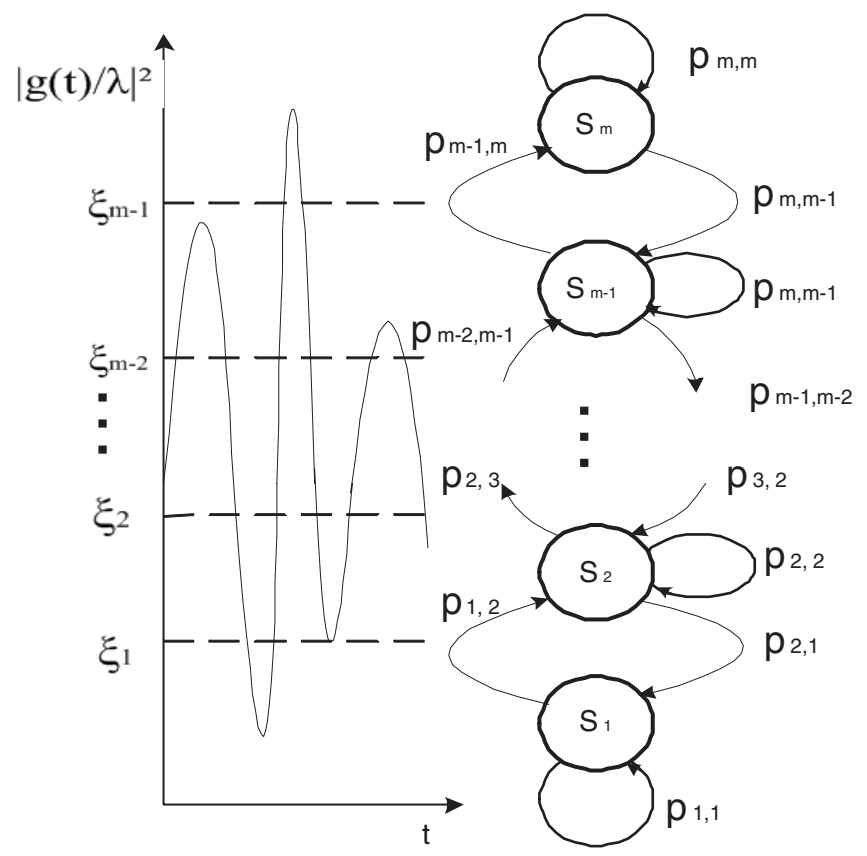

Fig. 3. Finite-state Markov model for fading channels

\section{SCHEDULING}

Implementation of a dedicated reverse channel is often criticized for practical complications and its possible negative impacts on spectrum utilization in communication systems. Furthermore, dedicated feedback channels seem to be redundant given that ARQ is traditionally implemented in wireless communication systems. Thus, the objective of this paper is to propose an alternative implementation of opportunistic scheduling solely relying on ARQ, i.e., ACK and NAK signals. The proposed schemes in this paper are implemented based on the estimation of channel state for individual users, given that the Markov models of the channels, as shown in Figure 3 and computed in Appendix , are available. The estimation of the channel states will be updated occasionally when the ACK or NAK signals for a particular user are available in order to avoid accumulation of estimation error. The scheduling schemes that are considered for performance analysis, which two of them are contributions of this paper, are described in the following.

\section{- Classical Opportunistic Scheduling (C-OS):}

For the classical opportunistic scheduling, a base station collects feedback information from users in each scheduling epoch. A mobile station estimates the current status of its channel from a pilot signal, computes a proper MCS, and reports the results back through a reverse channel to the base station. Given that a typical fading channel does not vary significantly over the next few time slots, the base station can determine the channel-state of a mobile station for the upcoming time slot with a very high precision (the decision procedure 
will be explained later in this section). The scheduler then selects only one mobile station with the highest achievable transmission rate in each time slot; a tie scenario, where more than one mobile station can achieve the highest transmission rate, is randomly broken. The transmitted data block is then removed from the buffer if successful reception is acknowledged by the mobile station.

\section{ARQ-based Opportunistic Scheduling with Explicit NAK (ARQ-OS-EN):}

Similar to C-OS, the scheduling decision is made based on the estimated CSI in each time slot. However, unlike C-OS, the scheduler does not have fresh feedback information from all of the mobile stations in every time slot. Instead, the base station uses the latest available estimation of the channel-state for each mobile station to decide its channel-state for the next time slot. Then, the scheduler randomly selects a single mobile station among those with the highest estimated MCS for transmission in the next time slot. Different from C-OS, in ARQOS-EN, the ACK/NAK messages for the selected user not only indicate a successful or unsuccessful reception of the recently transmitted packet, they also contain the latest channel-state report from the selected user, which is then used to update channel-state information of the base station about that particular user.

Note that an ARQ-OS-EN scheduler has less accurate CSI than a C-OS scheduler. Consequently, there will a higher probability of selecting a wrong user and wrong MCS, which will either cause a higher probability of unsuccessful transmission or under utilization of the available bandwidth. In other words, this scheme trades on an small portion of spectrum utilization to reduce system complexity. This seems however a reasonable trade-off, particularly, in the wireless networks without explicit feedback facility such as the IEEE 802.11 series.

\section{ARQ-based Opportunistic Scheduling with Implicit NAK (ARQ-OS-IN):}

Some implementation of link level retransmission, i.e., ARQ, schemes do not utilize explicit NAK messages to inform a sender node of an unsuccessful transmission. Instead, a sender node assumes an implicit NAK after a certain timeout period. Accordingly, ARQ-OS-IN relies only on the feedback information embedded in ACK messages. Unlike ARQ-OS-EN, an implicit NAK in ARQ-OS-IN indicates an unsuccessful transmission with no information about the actual channel-state. To reduce the risk of another erroneous transmission, the base station decrements its estimation of the channel-state of the tagged user.

\section{Scheduling without Feedback(S-NOF):}

This scheme is a simple Round Robin scheduling; each user is visited in a round and the MCS is chosen based on the average quality of channel. This scheme is only used as a benchmark to compare the performance of different feedback policies in opportunistic scheduling in this paper.

The channel-state of a mobile station for C-OS, ARQ-OSEN, and ARQ-OS-IN schemes is computed from the current channel-state of the mobile station as follows. If the current estimation of the channel-state of a tagged mobile station at time slot $t$, be $S_{i}$, the estimated channel-state at time $t+1$ will be $S_{j}$ with probability of $p_{i, j}$ for $j=1, \ldots, m$, where $m$ is the number of the states of the Markov model and $p_{i, j}$ is the $i, j^{t h}$ element of the probability transition matrix of the tagged mobile station.

\section{Simulations Results}

In this section, the Monte Carlo simulation settings for performance analysis are explained. Sample results and discussions are given to demonstrate the relative performance of the proposed approach in this paper.

A system model similar to Figure 2 is simulated by MATLAB in this paper. The envelope of Rayleigh fading channels are generated through filtering of two random white Gaussian processes as shown in Figure 4 , which is adopted from [11]. The Low Pass Filters (LPF) approximate the power spectrum density of fading process, given by

$$
S(f)= \begin{cases}\frac{\Omega_{p}}{4 \pi f_{m}} \frac{1}{\sqrt{1-\left(\frac{f-f_{c}}{f_{m}}\right)^{2}},}, & \left|f-f_{c}\right| \leq f_{m} \\ 0, & \text { otherwise, }\end{cases}
$$

where $f_{c}=1900 \mathrm{MHz}$ is the carrier frequency and $f_{m}$ is the maximum Doppler frequency shift. The time slot duration is $1.25 \mathrm{~ms}$, and the MCS threshold level set is $[0,2.5,5,7.5,10]$ dB. A saturated scenario is considered where there is always

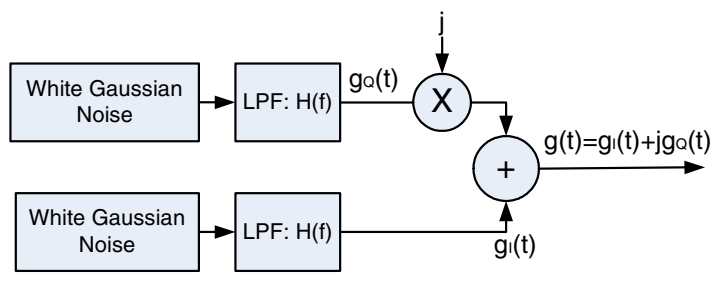

Fig. 4. Rayleigh fading channel simulator

buffered data for transmission in the base station. In the following, we present and discuss some sample results in order to compare the performance of the scheduling schemes that are described in Section III.

Figure 5 shows the total throughput of the different scheduling schemes versus the number of the active users. The results demonstrates that a scheduling scheme with explicit feedback channels, C-OS, outperforms the other scheduling schemes in terms of the total throughput. However, when there is no explicit feedback channels, comparison of the performance of C-NOF with ARQ-OS-IN and ARQ-OS-EN scheme shows 
that utilizing of the already existing ARQ messages can significantly improve the throughput of scheduling, withou having to embrace the complexity of independent feedback channels. As it can be seen in Figure 5, C-OS scheme can harvest the full multiuser diversity gain improving the system throughput with the increasing number of users. The results demonstrate that ARQ-OS-IN and ARQ-OS-EN schemes can also exploit multiuser diversity gain to a reasonable extent. The

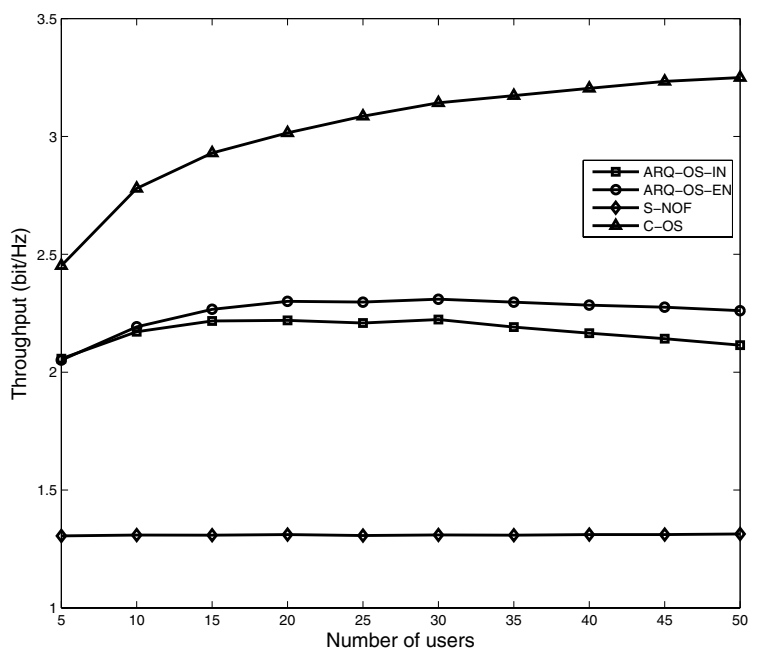

Fig. 5. The total throughput of the scheduler versus the number of users

impacts of increasing fading speed, i.e., maximum Doppler shift, on the total throughput of different scheduling schemes is shown in Figure 6. It can be seen that the increasing fading speed degrades the total system throughput of the opportunistic scheduling schemes, i.e., C-OS, ARQ-OS-IN, and ARQ-OS-EN schemes. A faster fading expectedly causes more estimation errors leading to a poorer throughput. Figure

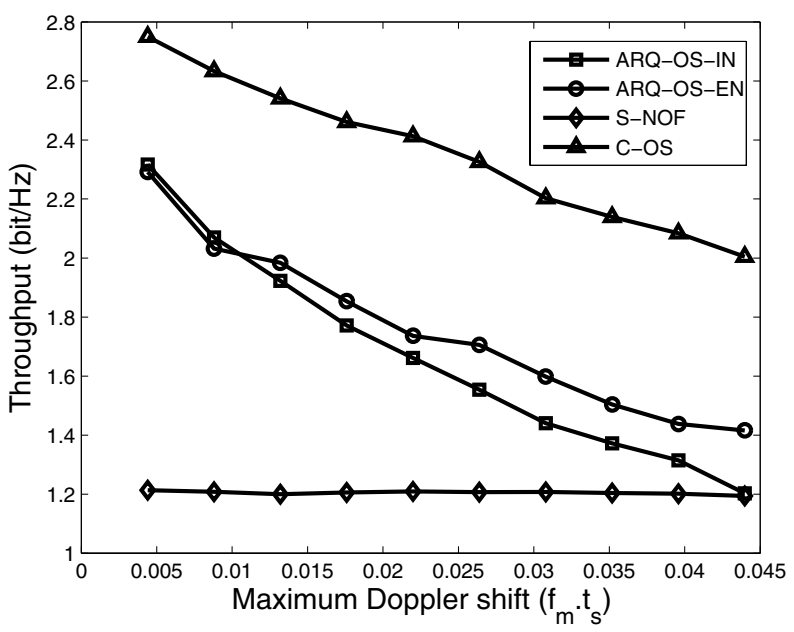

Fig. 6. The total throughput versus the fading speed
7 shows the Gini fairness index for the different scheduling schemes of Section III against the total number of users. Since the users have equal average channel quality and similar temporal dynamics, they demonstrate nearly perfect fairness as the fairness index is about .01 .

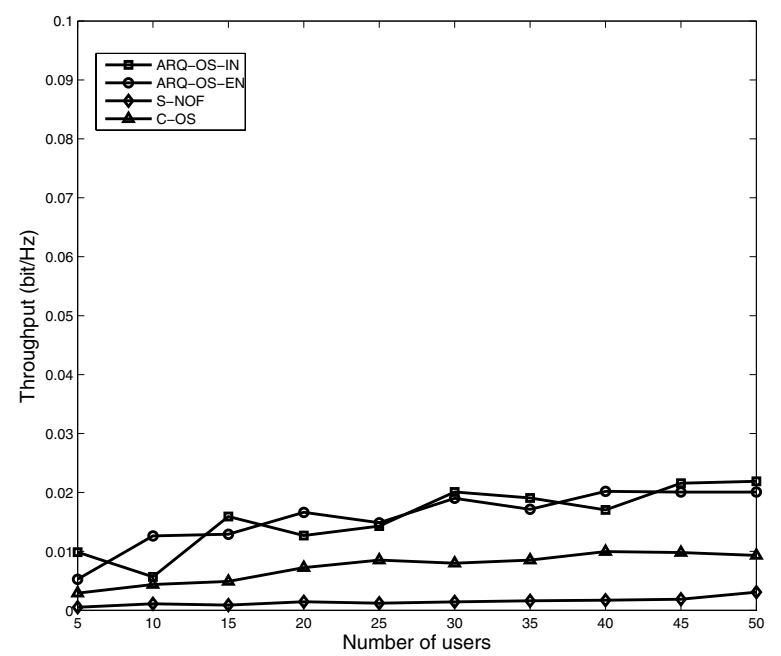

Fig. 7. Gini fairness index

\section{Conclusions}

In this paper, an alternative approach for implementation of opportunistic scheduling without explicit feedback channels is proposed. The results of our simulation based performance analysis demonstrates that the existing ARQ signals can be effectively used to achieve a significant throughput gain and exploit a reasonable level of multiuser diversity gain while avoiding the complexity of having explicit feedback channels.

\section{REFERENCES}

[1] R. Knopp and P. A. Humblet, "Information Capacity and Power Control in Single Cell Multiuser Communications," in Proc. of the IEEE Int. Conf. on Communications, June 1995, pp. 331-335.

[2] D. N. C. Tse, "Optimal Power Allocation over Parallel Gaussian Channels," in Proc. of Int. Symp. Information Theory, June 1997, pp. 27.

[3] H. Jahangir Hossain, M. S. Alouini, V. K. Bhargava, "Multi-User Opportunistic Scheduling using Power Controlled Hierarchical Constellations," IEEE Transactions on Wireless Communications, vol. 6, no. 5, May 2007, pp. 1581-1586.

[4] A. Farrokh, V. Krishnamurthy, "Opportunistic Scheduling for Streaming Multimedia Users in High-Speed Downlink Packet Access (HSDPA)," IEEE Transactions on Multimedia, vol. 8, no. 4, Aug. 2006, pp. 844-855.

[5] R. Casaquite, K. In-Yeup Kong, Y. Myung-Hyun Yoon, and H. WonJoo, "Opportunistic Scheduling with Power Control in Ad Hoc Wireless Networks," in Proc. of The 8th International Conference on Advanced Communication Technology, vol. 1, Feb. 2006, pp. 719-724.

[6] Y. Al-Harthi, A. Tewfik, and M. S. Alouini, "Opportunistic Scheduling with Quantized Feedback in Wireless Networks," in Proc. of Information Technology: Coding and Computing, 2005. (ITCC 2005), vol. 2, April 2005, pp. 716-722.

[7] S. H. Ali, V. Krishnamurthy, Victor C.M. Leung, "Optimal and Approximate Mobility-Assisted Opportunistic Scheduling in Cellular Networks," IEEE Transactions on Mobile Computing, vol. 6, no. 6, June 2007, pp. 633-648. 
[8] D. Gesbert and M. Slim-Alouini, "How much feedback is multi-user diversity really worth?," in Proc. IEEE International Conference on Communications, 2004, June 2004, pp. 234-238.

[9] Q. Ni, L. Romdhani, T. Turletti, "A Survey of QoS Enhancements for IEEE 802.11 Wireless LAN," Journal of Wireless Communications and Mobile Computing, Wiley, vol. 4, no. 5, 2004, pp. 547-566.

[10] V. Tykhomyrov, A. Sayenko, H. Martikainen, O. Alanen, T. Hmlinen, "Performance Evaluation of the IEEE 802.16 ARQ Mechanism," Lecture Notes in Computer Science, pringer Berlin, vol. 4712/2007, 2007, pp. 148-161.

[11] G.L. Stuber, "Principles of Mobile Communications," Kluwer Academic Publishers, 2001.

[12] M. Zorzi, R. R. Rao, and L. B. Milstein, "ARQ Error Control for Fading Mobile Radio Channels," IEEE Transactions on Vehicular Technology, vol. 46, no. 2, May 1997, pp. 445-455.

\section{APPENDIX}

Considering an isotropic propagation environment, where transmitted signal arrives at the receiver with a uniformly distributed angle from all directions, the fading envelope can be represented by a complex Gaussian process as $g(t)=$ $g_{I}(t)+j g_{Q}(t)$ [11]. where $g_{I}(t)$ and $g_{Q}(t)$ are two zeromean and white random Gaussian processes. The amplitude of fading envelope, $|g(t)|=\sqrt{g_{I}(t)^{2}+g_{Q}(t)^{2}}$, is therefore a non-white random process. It has been shown in [12] that a first-order Markov process can adequately model the dynamic of Rayleigh fading envelope. In this model, the channel is in good state $(\mathrm{G})$ if the normalized fading envelope power is above a certain threshold $\xi$, and in bad state (B), otherwise. The transition probabilities for Rayleigh fading model are given in [12] as

$$
v=\frac{Q(\theta, \rho \theta)-Q(\rho \theta, \theta)}{e^{\xi}-1}, u=\frac{1-e^{-\xi}}{e^{-\xi}} v,
$$

where $Q(\cdot, \cdot)$ is the Marcum $\mathrm{Q}$ function, $\theta=\sqrt{\frac{2 \xi}{1-\rho^{2}}}, \rho=$ $J_{0}\left(2 \pi f_{m} T\right)$. Note that $J_{0}(\cdot)$ is the zero-order Bessel function of the first kind, $f_{m}$ is the maximum Doppler frequency shift, and $T$ is the time span between two consecutive samples of the fading envelope. In our case, $T$ is equal to the length of a time slot.

Now, we extend the above two-state model into a m-state Markov model with arbitrary number of states, as illustrated in Figure 3. When the signal power is below $\xi_{i}$ and above $\xi_{i-1}$ the channel is in state $S_{i}$. Considering (2) with the threshold level $i$ yields a pair of transition probabilities as

$$
v_{i}=\frac{Q(\theta, \rho \theta)-Q(\rho \theta, \theta)}{e^{\xi_{i}}-1}, u_{i}=\frac{1-e^{-\xi_{i}}}{e^{-\xi_{i}}} v_{i},
$$

and $\xi_{i}=1-e^{-\xi_{i}}$, which represents the probability that the received normalized signal power, $|g(t)|^{2} / \Omega$, is below $\xi_{i}$. We define few primitives, $v_{i}, u_{i}, \xi_{i}$, which will be used for the rest of our computations. Let $s_{i}(t)$ represent the event that the channel is in state $i$ at time slot $t$. According to the basic definition of Markov models,

$$
\begin{aligned}
p_{i, i+1} & =\operatorname{Pr}\left\{s_{i+1}(t) \mid s_{i}(t-1)\right\} \\
& =\frac{\operatorname{Pr}\left\{s_{i+1}(t) \cap s_{i}(t-1)\right\}}{\operatorname{Pr}\left\{s_{i}(t-1)\right\}} .
\end{aligned}
$$

Hence, we need to find $\operatorname{Pr}\left\{s_{i+1}(t) \cap s_{i}(t-1)\right\}$ and $\operatorname{Pr}\left\{s_{i}(t-1)\right\}$ in order to compute $p_{i, i+1}$. From (3), we can directly conclude

$$
\operatorname{Pr}\left\{s_{i}(t-1)\right\}=\xi_{i}-\xi_{i-1} .
$$

To obtain $\operatorname{Pr}\left\{s_{i+1}(t) \cap s_{i}(t-1)\right\}$, first, we prove that $\operatorname{Pr}\left\{s_{i+1}(t) \cap s_{i}(t-1)\right\}=\operatorname{Pr}\left\{|g(t)|^{2} / \Omega \geq \xi_{i} \cap|g(t-1)|^{2} / \Omega \leq\right.$ $\left.\xi_{i}\right\}$. We expand the term on the right side as

$$
\begin{aligned}
& \operatorname{Pr}\left\{|g(t)|^{2} / \Omega \geq \xi_{i} \cap|g(t-1)|^{2} / \Omega \leq \xi_{i}\right\}= \\
& \operatorname{Pr}\left\{\left[s_{m}(t) \cup \cdots \cup s_{i+1}(t)\right] \cap\right. \\
& \left.\left[s_{i}(t-1) \cup \cdots \cup s_{1}(t-1)\right]\right\}= \\
& \operatorname{Pr}\left\{\left[\left[s_{m}(t) \cup \cdots \cup s_{i+1}(t)\right] \cap s_{i}(t-1)\right] \cup\right. \\
& \vdots \\
& \left.\left[\left[s_{m}(t) \cup \cdots \cup s_{i+1}(t)\right] \cap s_{1}(t-1)\right]\right\}= \\
& \operatorname{Pr}\left\{\left[\left(s_{m}(t) \cap s_{i}(t-1)\right) \cup \cdots \cup\left(s_{i+1}(t) \cap s_{i}(t-1)\right)\right] \cup\right. \\
& \vdots \\
& \left.\left[\left(s_{m}(t) \cap s_{1}(t-1)\right) \cup \cdots \cup\left(s_{i+1}(t) \cap s_{1}(t-1)\right)\right]\right\} .
\end{aligned}
$$

Recall from Figure 3 that only transitions among neighbor states are possible. Thus, any $\left(s_{k}(t) \cap s_{l}(t-1)\right)$ for $|k-l|>1$ is an empty set. Hence, all terms in (6), except $\left(s_{i+1}(t) \cap\right.$ $\left.s_{i}(t-1)\right)$, can be eliminated. In other words, $\operatorname{Pr}\left\{s_{i+1}(t) \cap\right.$ $\left.s_{i}(t-1)\right\}=\operatorname{Pr}\left\{|g(t)|^{2} / \Omega \geq \xi_{i} \cap|g(t-1)|^{2} / \Omega \leq \xi_{i}\right\}$. On the other hand, from the definition of transition probabilities for a two-state Markov model in (3),

$$
v_{i}=\frac{\operatorname{Pr}\left\{|g(t)|^{2} / \Omega \geq \xi_{i} \cap|g(t-1)|^{2} / \Omega \leq \xi_{i}\right\}}{\operatorname{Pr}\left\{s_{i}(t-1) \cup \cdots \cup s_{i}(t-1)\right\}}
$$

We have just proved that $\operatorname{Pr}\left\{s_{i+1}(t) \cap s_{i}(t-1)\right\}=$ $\operatorname{Pr}\left\{|g(t)|^{2} / \Omega \geq \xi_{i} \cap|g(t-1)|^{2} / \Omega \leq \xi_{i}\right\}$. In addition, $\operatorname{Pr}\left\{s_{i}(t-1) \cup \cdots \cup s_{i}(t-1)\right\}=\xi_{i}$. Hence, (7) can be simplified to

$$
v_{i}=\frac{\operatorname{Pr}\left\{s_{i+1}(t) \cap s_{i}(t-1)\right\}}{\xi_{i}} .
$$

Finally, combining (4), (5), and (8) yields

$$
p_{i, i+1}=\frac{\xi_{i}}{\xi_{i}-\xi_{i-1}} v_{i} \text {. }
$$

Similarly, we can show that

$$
p_{i+1, i}=\frac{1-\xi_{i}}{\xi_{i+1}-\xi_{i}} u_{i}
$$

Referring to Figure 3,

$$
p_{i, i}=1-p_{i, i+1}-p_{i, i-1} .
$$

